\title{
A Novel Distance-based Metric to Evaluate the Solution for Group Decision Making Problems under Consensus
}

\author{
Francisco J. Estrella ${ }^{1}$ Iván Palomares $^{2}$ Luis Martínez $^{1}$ \\ ${ }^{1}$ Computer Science Department, University of Jaén, Jaén (Spain) \\ ${ }^{2}$ Knowledge and Data Engineering Dept., Queens University of Belfast, Northern Ireland (United Kingdom)
}

\begin{abstract}
Consensus reaching processes play a key role in the resolution of many group decision making problems, in which it is desirable to obtain a highly acceptable solution to all individuals involved in the problem. However, not much interest has been put on defining appropriate metrics to evaluate in further detail the consensus solution, regarding experts' preferred ordering or ranking of alternatives. This contribution presents a metric based on Kemeny distance, that evaluates the consensus solution in terms of degree of satisfaction. Furthermore, based on this metric we aim at determining the extent to which applying a consensus reaching process improves the satisfaction with the solution.
\end{abstract}

Keywords: Group Decision Making, Consensus Reaching Process, Kemeny Distance, Preference Ordering.

\section{Introduction}

Decision making is a frequent daily activity in human lives, in which there exist several alternatives and we must decide which one/s should be chosen. In a Group Decision Making (GDM) problem, a group of experts with different interests attempt to make a common decision together $[1,2]$.

Classically, GDM problems have been solved by conducting an alternatives selection process only [3], in which the best alternative/s are chosen, often ranking alternatives from the best to the worst one. Nevertheless, some experts may not be satisfied with the solution made, hence they might disagree with it. Consensus Reaching Processes (CRPs) were introduced as an additional phase for the resolution of GDM problems, in order to overcome this drawback. In a CRP, experts iteratively revise and modify their initial preferences bringing them closer to each other, until reaching a high agreement level [4]. Consensus has become a major research topic in the field of GDM. A proof of this is the variety of consensus models proposed to support groups with CRPs in diverse GDM scenarios [5].

Despite the progress made in this topic of research, currently there are no appropriate tools to evaluate in further detail the result of applying a
CRP in terms of the experts' satisfaction with the solution achieved for the GDM problem. From a psychology point of view, one of the main factors that affect an individual's satisfaction is the perceived autonomy, in terms of his/her sense of selfelection and self-regard $[6,7]$. So, the closer the final GDM problem solution is to expert's initial opinion, the greater the feeling of autonomy, and therefore the more satisfied he/she will be with such a solution. Hence, it would be desirable to define a measure that reflects the level of experts' satisfaction with such a solution, with a focus on the most and least preferred alternatives by experts. Such a measure would allow not only to distinguish the degree of individual and collective satisfaction with the solution, but also to enable comparisons between the performance of different CRPs scenarios for the resolution of the same problem.

This contribution presents a distance-based metric to evaluate the solution for a GDM problem under consensus, regarding the level of expert and group satisfaction with such a solution. This work also shows how this metric can be utilized to determine the degree to with the CRP has improved the solution made, compared with the solution that would have been reached without applying a CRP. The metric proposed uses the Kemeny distance measure between individual and collective orderings of alternatives [8], therefore this work has particular interest in GDM problems in which the goal is obtaining a ranking of alternatives, instead of just choosing the best one.

This contribution is organized as follows: Section 2 reviews some preliminary concepts on GDM, CRPs and the Kemeny distance between orderings of alternatives. The distance-based metric to evaluate the consensus solution for GDM problems and its potential uses, are presented in Section 3. Section 4 shows an example of application of the metric, and finally some concluding remarks are drawn in Section 5.

\section{Preliminaries}

In this section, some basic concepts on GDM problems, CRPs and the Kemeny distance measure on orderings of alternatives, are briefly revised. 


\subsection{Group Decision Making}

GDM problems imply the participation of multiple experts, with different knowledge and interests, in a decision problem for which they have to find a common solution [1]. A GDM problem is formally composed by the following elements [2]:

(i) A group $E=\left\{e_{1}, \ldots, e_{m}\right\}$ of $m$ decision makers or experts.

(ii) A set $X=\left\{x_{1}, \ldots, x_{n}\right\}$ of $n$ alternatives or possible solutions to the problem.

Each expert $e_{i} \in E$ expresses his/her opinions over alternatives in $X$ by means of a preference structure. A commonly utilized preference structure in many GDM approaches is the fuzzy preference relation $[9,10]$. A fuzzy preference relation associated to $e_{i}$, denoted by $P_{i}=\left(p_{i}^{l k}\right)_{n \times n}$, can be represented for $X$ finite as follows:

$$
P_{i}=\left(\begin{array}{ccc}
- & \ldots & p_{i}^{1 n} \\
\vdots & \ddots & \vdots \\
p_{i}^{n 1} & \ldots & -
\end{array}\right)
$$

Each assessment, $p_{i}^{l k}=\mu_{P_{i}}\left(x_{l}, x_{k}\right) \in[0,1]$, represents $e_{i}$ 's degree of preference of $x_{l}$ with respect to $x_{k}, l, k \in\{1, \ldots, n\}, l \neq k$, such that:

- $p_{i}^{l k}>0.5$ means $e_{i}$ 's preference of $x_{l}$ over $x_{k}$.

- $p_{i}^{l k}<0.5$ means $e_{i}$ 's preference of $x_{k}$ over $x_{l}$.

- $p_{i}^{l k}=0.5$ means $e_{i}$ 's indifference between $x_{l}$ and $x_{k}$.

Elements $p_{i}^{l l}$ in the diagonal of the matrix are not elicited, hence they are denoted by "-".

Fuzzy preference relations can fulfill different properties [11], such as reciprocity, i.e. if $p_{i}^{l k}=x$, $x \in[0,1], l \neq k$, then $p_{i}^{k l}=1-x$. In order to deal with preferences under some degree of rationality and simplify the construction of fuzzy preference relations by experts, in this work we assume that experts must only provide $n-1$ assessments in the form $p_{i}^{l(l+1)} l=1, \ldots, n-1$. Thus, a consistent reciprocal fuzzy preference relation $P_{i}$ can be constructed upon these $n-1$ expert assessments, based on the method presented in [12].

The solution for a GDM problem can be obtained by applying either a direct approach or an indirect approach. In the former, the solution is directly obtained from experts' preferences, without constructing a social opinion first, whereas in the latter a social opinion or collective preference $P_{c}$ is previously computed and used to find the solution for the problem [3]. Regardless of the approach tackled, the classical alternative selection process for solving a GDM problem consists of two phases [13] (see Figure 1):

(i) Aggregation: In this phase, experts' preferences are combined by using an aggregation operator.

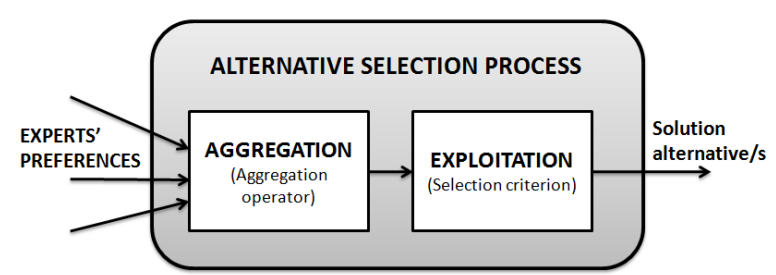

Figure 1: Selection process for the resolution of GDM problems

(ii) Exploitation: A selection criterion is applied to obtain an alternative or subset of them as the solution for the problem.

A well-known selection criterion for fuzzy preference relations is the non-dominance criterion [10], which computes for each alternative $x_{l} \in X$ a nondominance degree $N D\left(x_{l}\right) \in[0,1]$, as follows:

1. Construct a strict fuzzy preference relation $\tilde{P}_{i}=\left(\tilde{p}_{i}^{l k}\right)_{n \times n}$, where:

$$
\tilde{p}_{i}^{l k}=\left\{\begin{array}{cl}
p_{i}^{l k}-p_{i}^{k l} & \text { if } p_{i}^{l k}>p_{i}^{k l}, \\
0 & \text { otherwise }
\end{array}\right.
$$

2. Compute $N D\left(x_{l}\right)$ as:

$$
N D\left(x_{l}\right)=1-\max _{k}\left\{\tilde{p}_{i}^{k l}\right\}
$$

Alternatives are ranked from the best to the worst one based on $N D\left(x_{l}\right)$, such that $x_{l} \succ x_{k}$ iff $N D\left(x_{l}\right)>N D\left(x_{k}\right)$. From this ranking of alternatives, it can be obtained a preference ordering $O=\{o(1), \ldots, o(n)\}$, given by a permutation function $o(\cdot)$ over the index set $\{1, \ldots, n\}$. such that $o(l)=k$ means that $x_{l}$ is the $k$-ith best alternative.

\subsection{Consensus Reaching Processes}

Applying an alternatives selection process solely to solve a GDM problem, does not guarantee that there exists an agreement between experts before making the decision, since some experts might think that their opinions have not been heard sufficiently [14]. In order to overcome this drawback, CRPs were introduced as an additional phase in the resolution of GDM problems [4].

The concept of consensus has been subject of different interpretations in the literature, ranging from consensus as total agreement or unanimity, which is often difficult to achieve in practice, to more flexible interpretations. The notion of "soft consensus" based on fuzzy majority, proposed by Kacprzyk in [1], is one of the most accepted flexible notions of consensus, according to which there exist consensus in a group when "most of the important individuals agree as to (their testimonies concerning) almost all of the relevant options".

The CRP is an iterative and dynamic discussion process, in which experts modify their initial preferences bringing them closer to each other, with the 
aim of reaching a high level of collective agreement after several rounds [4]. This process is usually coordinated by a human figure called moderator, who is responsible for supervising and guiding the experts involved in the process. Figure 2 depicts a general CRP scheme followed by many approaches in the literature [5]. Its main phases are described below:

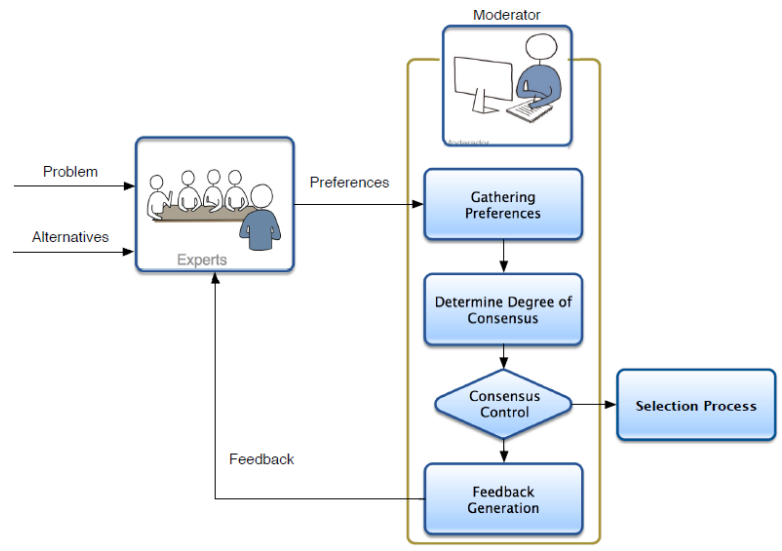

Figure 2: General CRP scheme

1. Gathering preferences: Each expert $e_{i}$ provides his/her preferences over alternatives to the moderator, e.g. by means of a fuzzy preference relation.

2. Determine degree of consensus: The current degree of consensus in the group is computed. To do this, different consensus measures can be utilized [5], based on measuring similarities/distances between preferences and the use of aggregation operators.

3. Consensus Control: The consensus degree is compared with a consensus threshold fixed a priori, which indicates the minimum level of agreement required. If the consensus degree exceeds such a threshold, then the group proceeds to the alternatives selection process to achieve a solution for the GDM problem; otherwise, it is necessary another discussion round. In order to limit the number of discussion rounds allowed, a parameter Maxround $\in \mathbb{N}$ can be introduced.

4. Feedback Generation: A collective preference, $P_{c}$, is computed by aggregating the individual preferences of all experts. Based on $P_{c}$, the farthest experts' assessments from consensus are identified, in order to advise them to modify such assessments with the aim of increasing the consensus degree in the following round.

\subsection{Kemeny Distance between Preference Orderings}

The proposal presented in this work computes distances between preference orderings over $X$, therefore it is necessary to choose a function that computes such distances. Here, we focus on the Ke- meny distance measure [8], which computes the distance between two preference orderings $O_{i}=$ $\left\{o_{i}(1), \ldots, o_{i}(n)\right\}$ and $O_{j}=\left\{o_{j}(1), \ldots, o_{j}(n)\right\}$ based on the number of pairs of elements (in our case, alternative rankings $\left.o_{i}(l) \in\{1, \ldots, n\}\right)$ whose relative ranking differs between $O_{i}$ and $O_{j}$.

Firstly, let $o_{i j}: \mathbb{N} \times \mathbb{N} \rightarrow[0,1]$ be a function defined over pairs of alternative rankings in two preference orderings, such that:

$o_{i j}(l, k)=\left\{\begin{array}{cc}1 & \text { if }\left(o_{i}(l)<o_{i}(k) A N D o_{j}(l)>o_{j}(k)\right), \\ 1 & \text { if }\left(o_{i}(l)>o_{i}(k) A N D o_{j}(l)<o_{j}(k)\right), \\ 0.5 & \text { if }\left(o_{i}(l)=o_{i}(k) A N D o_{j}(l) \neq o_{j}(k)\right), \\ 0.5 & \text { if }\left(o_{i}(l) \neq o_{i}(k) A N D o_{j}(l)=o_{j}(k)\right), \\ 0 & \text { otherwise. }\end{array}\right.$

This function returns 1 if the two orderings show opposite preferences between $x_{l}$ and $x_{k}$, it returns 0.5 if one (and only one) of the two orderings shows indifference between both alternatives, and returns 0 otherwise (i.e. when both orderings reflect the same type of preference between $x_{l}$ and $x_{k}$ ).

Based on $o_{i j}$, the Kemeny distance between two preference orderings, denoted by $K\left(O_{i}, O_{j}\right)$, can be computed as follows [15]:

$$
K\left(O_{i}, O_{j}\right)=\sum_{l=1}^{n-1} \sum_{k=l+1}^{n} o_{i j}\left(x_{l}, x_{k}\right)
$$

with $K\left(O_{i}, O_{j}\right) \in\left[0, \frac{n(n-1)}{2}\right] . \quad K$ satisfies the following general properties of distance functions $K$ : $A \times A \rightarrow \mathbb{R}$, for any $a, b, c \in A[16]$ :

i) $K(a, b) \geq 0$ (non-negativity).

ii) $K(a, b)=0 \Leftrightarrow a=b$ (identity or indiscernibles).

iii) $K(a, b)=K(b, a)$ (symmetry).

iv) $K(a, b) \leq K(a, c)+K(c, b)$ (triangle inequality).

Although $K\left(O_{i}, O_{j}\right)$ takes values in the interval $\left[0, \frac{n(n-1)}{2}\right]$, we consider normalizing it to take a value in the unit interval:

$$
\bar{K}\left(O_{i}, O_{j}\right)=\frac{K\left(O_{i}, O_{j}\right)}{\frac{n(n-1)}{2}}=\frac{2 \cdot K\left(O_{i}, O_{j}\right)}{n(n-1)}
$$

\section{A Distance-based Metric to Evaluate the Consensus Solution for GDM Problems}

This section introduces a novel metric based on Kemeny distance to assess the solution obtained for a GDM problem involving a CRP. The main goal of the metric proposed here, is to define a more descriptive means to evaluate the goodness of a GDM problem solution than the final consensus degree achieved, which has often been used to measure how good such a solution is. This proposal considers the goodness or satisfaction degree of a problem solution in terms of its closeness to individual preferences of experts, $P_{i}$. The consensus solution for the problem is given by the ranking of alternatives obtained from $P_{c}$ after reaching consensus. 
Thus, the metric proposed aims at:

(1) Evaluating the degree of experts' satisfaction with the final solution made at an individual and collective level, based on their individual ranking of preferred alternatives at the end of a CRP.

(2) Determining the degree to which the CRP applied has improved the solution obtained, with respect to having applied a selection process only [3]. To do this, we propose comparing the degree of satisfaction with the solution that would be obtained before and after conducting the CRP.

(3) Providing a means to compare the solutions achieved by using different consensus models for the same problem, or a single consensus model under different settings of its parameters (see Section 4). Thus, it is possible to discern which solution is better even in the cases that all of them present a similar consensus degree.

Remark 1 Although the term "metric" is usually regarded as a synonymous of distance [16], in the scope of this proposal metric is understood as " $a$ standard of measurement".

Remark 2 Without loss of generality, this work focuses on the use of fuzzy preference relations, but the underlying ideas in our proposal are extendable to other types of preferences from which a ranking of alternatives can be obtained.

Consider a CRP in which consensus has been achieved after $T \in \mathbb{N}$ rounds. Hereinafter, let us denote an expert $e_{i}$ 's final preferences at the end of a $\mathrm{CRP}$ as $P_{i}^{T}$, and its corresponding ordering of alternatives as $O_{i}^{T}$. Similarly, an expert's initial preference and ordering of alternatives at the beginning of a CRP shall be denoted by $P_{i}^{1}$ and $O_{i}^{1}$, respectively.

The following metric is defined at both individual and group level, based on the difference between experts' modified preferences at the end of the CRP and the consensus solution achieved for the GDM problem.

Definition 1 Let $\bar{K}\left(O_{i}^{T}, O_{c}^{T}\right)$ be the Kemeny-based distance between an expert final preference ordering, $O_{i}^{T}$, and the collective final preference ordering, $O_{c}^{T}$. A metric indicating the individual or subjective satisfaction degree of $e_{i}$ with the solution is then defined as:

$$
Q_{i}=1-\bar{K}\left(O_{i}^{T}, O_{c}^{T}\right)
$$

with $Q_{i} \in[0,1]$.

Figure 3 illustrates graphically the computation of the individual satisfaction degree with the solution, $Q_{i}$.
Definition 2 Let $E=\left\{e_{1}, \ldots, e_{m}\right\}$. A metric $Q$ that indicates the group or objective satisfaction with the solution made is defined by:

$$
Q=\phi\left(Q_{1}, Q_{2}, \ldots, Q_{m}\right)
$$

with $Q \in[0,1]$ and $\phi:[0,1]^{m} \rightarrow[0,1]$ being an aggregation function.

This quality metric indicates how close experts' preferences are to the consensus solution made, based on their corresponding orderings of alternatives.

In the two definitions above, the higher $Q_{i}$ and $Q$, the more satisfied the expert or the group would be with the solution, respectively, since the higher the similarity between their preferred rankings of alternatives and $O_{i}^{T}$.

The choice of the aggregation function $\phi$ to combine individual satisfaction values $Q_{i}$ into an overall satisfaction $Q$, may depend on the attitude we want to reflect in such an aggregation [17]. For example, the OWA (Ordered Weighted Averaging) operator [18] could be used to give more importance to higher or lower individual satisfaction degrees in the aggregation process.

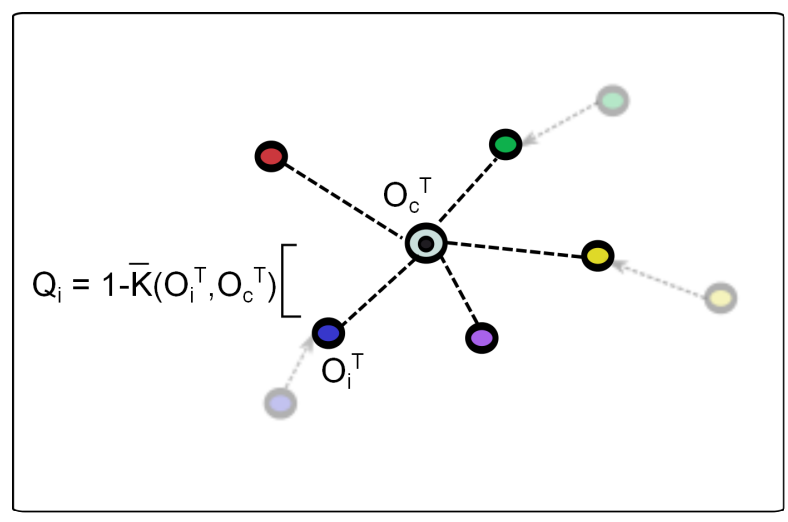

Figure 3: Graphical illustration of experts' satisfaction degree with the solution obtained

An example of computation of this metric at a subjective and objective level is given below:

Example 1 Consider a GDM problem consisting of four alternatives $(n=4)$, in which four experts are involved $(m=4)$. Each expert $e_{i}$ expresses his/her preferences over alternatives by means of a reciprocal consistent fuzzy preference relation $P_{i}$. A CRP is carried out with a consensus threshold $\mu=0.85$, and consensus is achieved at the sixth round, i.e. $T=6$.

After applying a selection process on the final collective preference $P_{c}^{T}$, the following solution is obtained: $O_{c}^{T}=\{3,4,2,1\}$, whose equivalent ranking of alternatives is:

$$
x_{4} \succ x_{3} \succ x_{1} \succ x_{2}
$$

Table 1 shows the final ordering of alternatives $O_{i}^{T}$ and its equivalent ranking for each expert at the end 
of the CRP, and the individual degree of satisfaction with the solution, $Q_{i}$.

Table 1: Distances between final experts' orderings of alternatives and the final group ordering

\begin{tabular}{|c|c|c|c|}
\hline$e_{i}$ & $O_{i}^{T}$ & Ranking & $Q_{i}$ \\
\hline$e_{1}$ & $\{3,2,1,4\}$ & $x_{3} \succ x_{2} \succ x_{1} \succ x_{4}$ & 0.33 \\
\hline$e_{2}$ & $\{4,2,3,1\}$ & $x_{4} \succ x_{2} \succ x_{3} \succ x_{1}$ & 0.67 \\
\hline$e_{3}$ & $\{4,3,2,1\}$ & $x_{4} \succ x_{3} \succ x_{2} \succ x_{1}$ & 0.83 \\
\hline$e_{4}$ & $\{3,2,4,1\}$ & $x_{4} \succ x_{2} \succ x_{1} \succ x_{3}$ & 0.5 \\
\hline
\end{tabular}

Assuming the use of a OWA operator with weights $W=\{0.3,0.3,0.2,0.2\}$ [18], the group satisfaction degree, $Q$, is computed as follows:

$Q=0.3 \cdot \boldsymbol{0 . 8 3}+0.3 \cdot \boldsymbol{0 . 6 7}+0.2 \cdot \boldsymbol{0 . 5}+0.2 \cdot \boldsymbol{0 . 3 3}=0.617$

We have defined a Kemeny-distance metric for determining individual (subjective) and group (objective) satisfaction with a consensus solution for a GDM problem. In the following, we also aim at determining the degree to which such a solution is better than the solution that would have been obtained in the case of not having conducted a CRP. This "non-consensus" solution can be derived from the initial collective preference $P_{c}^{1}$ (which is computed by aggregating the initial experts' preferences $\left.P_{i}^{1}\right)$. Thus, by calculating the difference between the value of $Q$ (resp. $Q_{i}$ ) at the beginning and end of the CRP, it is possible to determine the variation in the individual/group satisfaction degree, respectively, i.e. the degree to which the GDM problem solution has improved as a result of the CRP.

Definition 3 Let $Q_{i}^{1}$ be computed as follows:

$$
Q_{i}^{1}=1-\bar{K}\left(O_{i}^{1}, O_{c}^{1}\right)
$$

Similarly, let $Q^{1}$ be computed as:

$$
Q^{1}=\phi\left(Q_{1}^{1}, Q_{2}^{1}, \ldots, Q_{m}^{1}\right)
$$

being $\phi$ the same aggregation function used to compute $Q$ (see Eq.(4)). The individual or subjective degree of improvement in the consensus solution for $e_{i}$, is then obtained as follows:

$$
\Delta\left(Q_{i}\right)=Q_{i}-Q_{i}^{1}
$$

and the group or objective degree of improvement in such a solution is computed as:

$$
\Delta(Q)=Q-Q^{1}
$$

Example 2 Considering again the GDM problem formulated in Example 1, and assuming that the initial collective preference $P_{c}^{1}$ leads to the following solution before applying a CRP, $O_{c}^{1}=\{3,2,4,1\}$, i.e.:

$$
x_{4} \succ x_{2} \succ x_{1} \succ x_{3}
$$

Table 2 shows the initial ordering of alternatives $O_{i}^{1}$ of each expert before initiating the CRP, and the individual degree of satisfaction that they would have with the non-consensus solution shown above.

Table 2: Distances between initial experts' orderings of alternatives and the initial group solution

\begin{tabular}{|c|c|c|c|}
\hline$e_{i}$ & $O_{i}^{1}$ & Ranking & $Q_{i}^{\perp}$ \\
\hline$e_{1}$ & $\{1,2,3,4\}$ & $x_{1} \succ x_{2} \succ x_{3} \succ x_{4}$ & 0.33 \\
\hline$e_{2}$ & $\{4,2,3,1\}$ & $x_{4} \succ x_{2} \succ x_{3} \succ x_{1}$ & 0.83 \\
\hline$e_{3}$ & $\{4,3,2,1\}$ & $x_{4} \succ x_{3} \succ x_{2} \succ x_{1}$ & 0.67 \\
\hline$e_{4}$ & $\{3,2,1,4\}$ & $x_{3} \succ x_{2} \succ x_{1} \succ x_{4}$ & 0.17 \\
\hline
\end{tabular}

By using the same OWA operator considered previously, $Q^{1}$ is computed:

$Q^{1}=0.3 \cdot \boldsymbol{O} .83+0.3 \cdot \boldsymbol{0 . 6 7}+0.2 \cdot \boldsymbol{0 . 3 3}+0.2 \cdot \boldsymbol{0 . 1 7}=0.55$

Therefore, we have that $\Delta(Q)=0.617-0.55=$ 0.067 i.e. since $\Delta(Q)>0$ the solution has been slightly improved by applying the CRP, under a collective point of view. However, if we compute $\Delta\left(Q_{i}\right)$ for each expert (based on Tables 1 and 2), we can see that only $e_{3}$ and $e_{4}$ satisfaction with the solution has increased, whereas $e_{1}$ is equally satisfied with the consensus and non-consensus solution, and $e_{2}$ is less satisfied with the consensus solution (because $\left.\Delta\left(Q_{2}\right)<0\right)$. This result led us to conclude that, even though as a result of a CRP the overall group satisfaction with the solution may increase, in certain cases some experts' preferences might become further from the final solution made.

\section{Illustrative Example}

In order to analyze the usefulness and performance of the metric proposed in Section 3, in this section we present two examples based on the simulation of CRPs for the resolution of GDM problems.

For this purpose, we have defined a GDM problem characterized by a group of eight experts, $E=$ $\left\{e_{1}, e_{2}, e_{3}, e_{4}, e_{5}, e_{6}, e_{7}, e_{8}\right\}$, and a set of six alternatives, $X=\left\{x_{1}, x_{2}, x_{3}, x_{4}, x_{5}, x_{6}\right\}$, under which the CRPs take place.

At the beginning of the CRP, the ranking of alternatives taken from $P_{c}^{1}$ is $x_{1} \succ x_{2} \succ x_{3} \succ x_{4} \succ$ $x_{5} \succ x_{6}$, and the initial consensus degree, denoted by $C D \in[0,1]$, has a value of 0.59 . Initial experts' preference orderings are summarized in Table 3, together with the values of $Q_{i}^{1}$ (individual satisfaction with the non-consensus solution) for each expert.

Taking $Q_{i}^{1}$ values from Table 3 , and using the arithmetic mean as the aggregation operator $\phi$, the initial group satisfaction degree $Q^{1}$, is computed as 0.53. This value will be later used to calculate the group degree of improvement in the consensus solution $\Delta(Q)$.

The same consensus model is used in both simulations, which differ from each other in the patterns of behavior adopted by experts regarding the 
Table 3: CRP initial values

\begin{tabular}{|c|c|c|}
\hline$e_{i}$ & $O_{i}^{1}$ & $Q_{i}^{1}$ \\
\hline$e_{1}$ & $\{6,5,3,4,2,1\}$ & 0.07 \\
\hline$e_{2}$ & $\{3,2,1,4,5,6\}$ & 0.8 \\
\hline$e_{3}$ & $\{1,3,2,5,4,6\}$ & 0.87 \\
\hline$e_{4}$ & $\{5,6,2,3,4,1\}$ & 0.27 \\
\hline$e_{5}$ & $\{2,3,5,1,4,6\}$ & 0.73 \\
\hline$e_{6}$ & $\{1,3,2,4,5,6\}$ & 0.93 \\
\hline$e_{7}$ & $\{4,3,5,2,1,6\}$ & 0.47 \\
\hline$e_{8}$ & $\{5,6,4,2,3,1\}$ & 0.13 \\
\hline
\end{tabular}

way they accept the recommendations received in the advice generation phase and modify their preferences following such recommendations. Once the CRPs for the two simulations are conducted based on the GDM problem described above, in the following subsections we analyze the data obtained from each simulation, both of which were performed with a consensus threshold $\mu$ set at 0.95 . In this analysis, we will review the different values of $Q$ and $\Delta(Q)$ in each of the simulations.

\subsection{Simulation 1}

In the first simulation, we assume a scenario in which the experts are highly receptive to the recommendations received from the moderator, such that consensus should be reached after a small number of rounds.

The Simulation 1 ends after eight rounds, reaching a consensus degree of 0.95 .

The group preference ordering $O_{c}^{T}$, Consensus Degree at round $\mathrm{T}, C D^{T}$, and the resulting group metric value $Q^{T}$ and $\Delta(Q)^{T}$ for each round, are shown in Table 4. Notice here that, for the sake of enabling a more detailed analysis over the course of the CRP, we also compute the values $Q^{T}$ and $\Delta(Q)^{T}$ that would have been obtained if the CRP had ended at earlier rounds.

Table 4: Results per round in Simulation 1

\begin{tabular}{|c|c|c|c|c|}
\hline$T$ & $O_{c}^{T}$ & $C D^{T}$ & $Q^{T}$ & $\Delta(Q)^{T}$ \\
\hline 0 & $\{1,2,3,4,5,6\}$ & 0.59 & 0.53 & \\
\hline 1 & $\{3,5,4,2,1,6\}$ & 0.63 & 0.51 & -0.02 \\
\hline 2 & $\{3,5,2,4,1,6\}$ & 0.67 & 0.52 & -0.01 \\
\hline 3 & $\{3,5,2,1,4,6\}$ & 0.72 & 0.53 & 0 \\
\hline 4 & $\{3,5,2,1,4,6\}$ & 0.8 & 0.55 & 0.02 \\
\hline 5 & $\{3,5,2,1,4,6\}$ & 0.83 & 0.59 & 0.06 \\
\hline 6 & $\{3,2,1,5,4,6\}$ & 0.87 & 0.61 & 0.08 \\
\hline 7 & $\{3,2,1,5,4,6\}$ & 0.92 & 0.68 & 0.15 \\
\hline 8 & $\{3,2,5,1,4,6\}$ & 0.95 & $\mathbf{0 . 7 4}$ & $\mathbf{0 . 2 1}$ \\
\hline
\end{tabular}

The analysis of Table 4 shows the fact that the value of $Q^{T}$ decreases slightly in the first round ($0.02)$ from its initial value $(0.53)$, and then it incre- ments its value steadily in each of the rounds up to a maximum value of 0.74 in the final round, which results in $\Delta(Q)^{T}=0.21$.

The results shown above are graphically depicted in Figure 4, which represents the values of $C D^{T}$, $Q^{T}$ and $\Delta(Q)^{T}$ for each of the rounds.

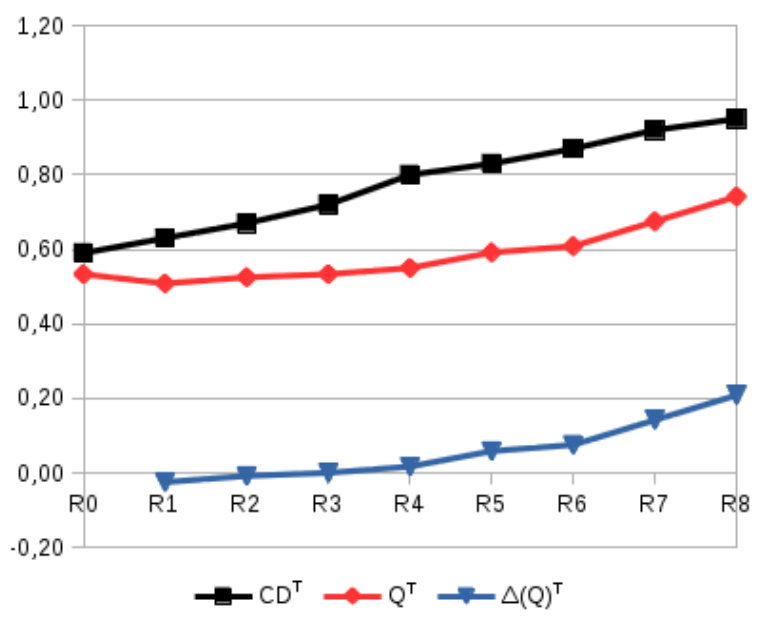

Figure 4: Results of Simulation 1

\subsection{Simulation 2}

In the second simulation, we assume a different scenario in which the experts are mostly reluctant to accept the recommendations. In this case, the number of rounds required to reach the set consensus threshold $\mu=0.95$, is greater. Consensus is achieved after fifteen rounds with a consensus degree of 0.96 .

Table 5 shows the values obtained in the CRP for each of the fifteen rounds carried out.

Table 5: Results per round in Simulation 2

\begin{tabular}{|c|c|c|c|c|}
\hline$T$ & $O_{c}^{T}$ & $C D^{T}$ & $Q^{T}$ & $\Delta(Q)^{T}$ \\
\hline 0 & $\{1,2,3,4,5,6\}$ & 0.59 & 0.53 & \\
\hline 1 & $\{3,5,4,2,1,6\}$ & 0.63 & 0.53 & 0 \\
\hline 2 & $\{3,5,1,2,4,6\}$ & 0.68 & 0.51 & -0.02 \\
\hline 3 & $\{3,5,1,2,4,6\}$ & 0.69 & 0.53 & 0 \\
\hline 4 & $\{3,5,1,2,4,6\}$ & 0.74 & 0.53 & 0 \\
\hline 5 & $\{3,5,2,1,4,6\}$ & 0.77 & 0.53 & 0 \\
\hline 6 & $\{3,1,2,5,4,6\}$ & 0.81 & 0.61 & 0.08 \\
\hline 7 & $\{3,5,2,1,4,6\}$ & 0.82 & 0.59 & 0.06 \\
\hline 8 & $\{3,5,2,1,4,6\}$ & 0.84 & 0.61 & 0.08 \\
\hline 9 & $\{3,2,5,1,4,6\}$ & 0.87 & 0.76 & 0.23 \\
\hline 10 & $\{3,2,5,1,4,6\}$ & 0.87 & 0.75 & 0.22 \\
\hline 11 & $\{3,2,5,1,4,6\}$ & 0.88 & 0.81 & 0.28 \\
\hline 12 & $\{3,2,5,1,4,6\}$ & 0.91 & 0.81 & 0.28 \\
\hline 13 & $\{3,2,5,1,4,6\}$ & 0.91 & 0.81 & 0.28 \\
\hline 14 & $\{3,2,5,1,4,6\}$ & 0.94 & 0.8 & 0.27 \\
\hline 15 & $\{3,2,5,1,4,6\}$ & 0.96 & $\mathbf{0 . 8}$ & $\mathbf{0 . 2 7}$ \\
\hline
\end{tabular}

In view of the data of Table 5 , it is reflected that 
the value of $Q^{T}$ remains stable until the fifth round. After that, the value of $Q^{T}$ starts to increase reaching its maximum value at the eleventh round with a value of $Q^{T}=0.81$ and $\Delta(Q)^{T}=0.28$ (see Figure 5). Once $Q^{T}$ reaches its maximum value, then it remains stable until the end of the CRP, only slightly decreasing its value at the 14 -ith round in 0.01 .

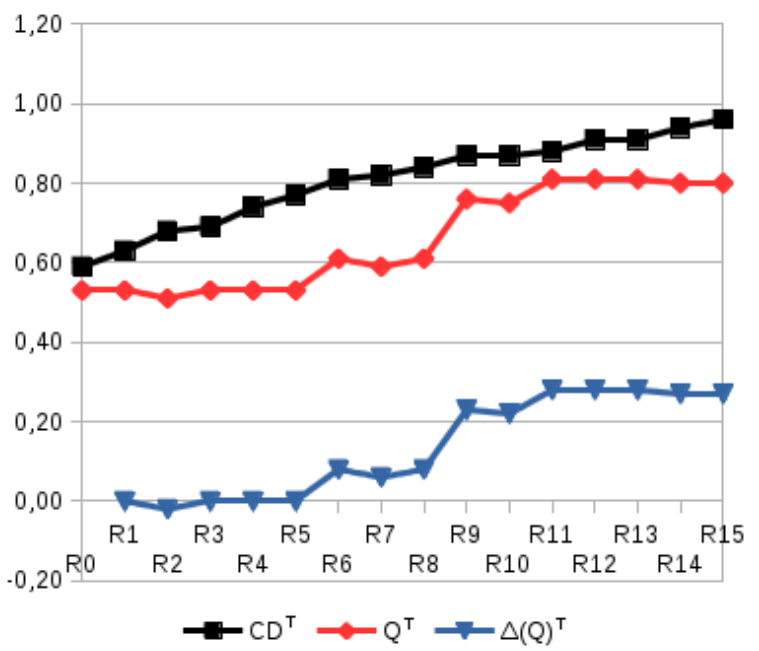

Figure 5: Results of Simulation 2

\subsection{Analysis of results}

Results of the example simulations show that $Q$ and, consequently $\Delta(Q)$, tends to increase in value during the CRP. This reflects not only that the CRP contributes to achieve a greater degree of consensus amongst experts, but also that the result is more satisfactory to them after conducting the process.

Regarding the group satisfaction degrees $Q$ observed for the different rounds in both example simulations, $Q^{T}$, they described an increase in value more pronounced in the intermediate rounds of the process, showing on the other hand a more stable trend in the initial and final rounds.

It should be pointed out that in both cases, $Q^{T}$ value begins to increase when the CRP have reached similar consensus degrees (0.80 in Simulation 1 and 0.81 in Simulation 2) and although in Simulation 1 the value of $Q^{T}$ keeps increasing until the end of the process, the maximum value achieved in this simulation, 0.74, is exceeded in Simulation 2 with a value of 0.81 .

Considering the differences in experts' behavior in the simulations performed we can see how, if the experts' behavior is more cooperative to reach consensus (Simulation 1), $Q^{T}$ almost follows a linear function, whereas if experts' behaviour is less favorable to reach consensus (Simulation 2), the value of $Q^{T}$ becomes more difficult to estimate.

\section{Concluding Remarks}

Given the inherent difficulty in Group Decision Making problems that all experts reach an agreement on the solution made, it is necessary to apply a consensus reaching process as an additional step for the resolution of such problems, aimed at finding the best solution according to the preferences of all the experts. Despite the extensive research in this area, we find that the definition of appropriate metrics to evaluate in depth the goodness or experts satisfaction with the solution obtained has not been sufficiently addressed. In this contribution, we have presented a novel Kemeny distance-based metric to evaluate, based on the expert and group satisfaction, the solution for a GDM problem under consensus. Furthermore, we have performed an initial experimental study to analyze the behavior of this metric in different scenarios and showed the results of such a study in an illustrative example.

This work is at an initial stage of development, hence there are multiple future directions for work that we have considered, such as: (i) studying the behavior of the proposed metric in different consensus models, (ii) taking into consideration the cost invested by experts in modifying their initial opinions for the computation of their degree of satisfaction with the consensus solution, or (iii) using weighted distance-based metrics which regard the positions of alternatives in the ranking, amongst others.

\section{Acknowledgements}

Financial support provided by the Doctorate School of the University of Jaén (EDUJA), Research Project TIN-2012-31263 and RDF.

\section{References}

[1] J. Kacprzyk. Group decision making with a fuzzy linguistic majority. Fuzzy Sets and Systems, 18(2):105-118, 1986.

[2] J. Lu, G. Zhang, D. Ruan, and F. Wu. MultiObjective Group Decision Making. Imperial College Press, 2006.

[3] F. Herrera, E. Herrera-Viedma, and J. Verdegay. A sequential selection process in group decision making with linguistic assessments. Information Sciences, 85(1995):223-239, 1995.

[4] S. Saint and J. R. Lawson. Rules for Reaching Consensus. A Modern Approach to Decision Making. Jossey-Bass, 1994.

[5] I. Palomares, F.J. Estrella, L. Martínez, and F. Herrera. Consensus under a fuzzy context: Taxonomy, analysis framework AFRYCA and experimental case of study. Information $\mathrm{Fu}$ sion, 20(November 2014):252-271, 2014.

[6] R. De Charms. Personal causation: The internal affective determinants of behavior. Routledge, 2013. 
[7] K. Sheldon, R. Ryan, and H. Reis. What makes for a good day? competence and autonomy in the day and in the person. Personality and Social Psychology Bulletin, (22):1270-1279, 1996.

[8] J. Kemeny. Mathematics without numbers. Daedalus, 88:571-591, 1959.

[9] R.O. Parreiras, P. Ekel, and F. Bernardes Jr. A dynamic consensus scheme based on a nonreciprocal fuzzy preference relation modeling. Information Sciences, 211(1):1-17, 2012.

[10] S.A. Orlovsky. Decision-making with a fuzzy preference relation. Fuzzy Sets and Systems, 1(3):155-167, July 1978.

[11] J. Fodor and M. Roubens. Fuzzy Preference Modelling and Multicriteria Decision Support. Kluwer, Dordrecht, 1994.

[12] F. Chiclana, E. Herrera-Viedma, S. Alonso, and F. Herrera. Cardinal consistency of reciprocal preference relations: a characterization of multiplicative transitivity. IEEE Transactions on Fuzzy Systems, 17(1):14-23, 2009.

[13] M. Roubens. Fuzzy sets and decision analysis. Fuzzy Sets and Systems, 90(2):199-206, 1997.

[14] C.T.L. Butler and A. Rothstein. On Conflict and Consensus: A Handbook on Formal Consensus Decision Making. Takoma Park, 2006.

[15] Mohamed Drissi-Bakhkhat and Michel Truchon. Maximum likelihood approach to vote aggregation with variable probabilities. Social Choice and Welfare, 23(2):161-185, 2004.

[16] M. Deza and E. Deza. Encyclopedia of Distances. Springer, 2014.

[17] G. Beliakov, A. Pradera, and T. Calvo. Aggregation Functions: A Guide for Practitioners. Springer, 2007.

[18] R.R. Yager. On orderer weighted averaging aggregation operators in multi-criteria decision making. IEEE Transactions on Systems, Man and Cybernetics, 18(1):183-190, 1988. 